\title{
Torus mandibularis in skeletal remains from the Joseon Dynasty and modern Korea
}

\author{
Helen $\mathrm{CHO}^{1 *}$, Dong Hoon $\mathrm{SHIN}^{2}$, Yisuk KIM${ }^{3}$, Heejin $\mathrm{KIM}^{4}$, Uyoung LEE \\ ${ }^{1}$ Department of Anthropology, Davidson College, North Carolina, USA \\ ${ }^{2}$ Department of Anatomy, Seoul National University College of Medicine, Seoul, Korea \\ ${ }^{3}$ Department of Anatomy, Ewha Womans University School of Medicine, Seoul, Korea \\ ${ }^{4}$ Department of Oral Biology, College of Dentistry, Yonsei University, Seoul, Korea \\ ${ }^{5}$ Department of Anatomy, Catholic University College of Medicine, Seoul, Korea
}

Received 9 May 2017; accepted 3 November 2017

\begin{abstract}
Torus mandibularis (TM), or mandibular tori, are nodular, excessive bony growths along the lingual side of the lower jaw. TM occurs in varying frequencies in different populations with a higher prevalence in Asiatic and circumpolar groups. The tori are associated with mechanical stress from masticatory and paramasticatory behaviors, heredity, sex, age, and environmental factors. We investigated the presence, location, symmetry, and degree of TM frequencies in two skeletal populations with cultural, biological, and geographical affinities: 15th-18th-century Joseon Dynasty Koreans $(n=119)$ and 20th-21st-century modern Koreans $(n=77)$. TM frequency is significantly higher among modern Koreans $(54.6 \%)$ than Joseon Dynasty individuals (13.3\%) for females and males. There are no sex differences in TM prevalence in the archaeological and modern groups and an age-related trend in the TM frequency is not observed in either sample. Our results are consistent with the literature, which reports a higher frequency in Asiatic populations, but contrary to the general worldwide trend of decreasing prevalence over time. Modern Koreans have not undergone significant dietary changes since the Joseon Dynasty, and the quasicontinuous model with external factors may be responsible for the development of TM.
\end{abstract}

Key words: mandible, torus, exostosis, Joseon, Korea

\section{Introduction}

Mandibular tori, or torus mandibularis (TM), are localized exostoses, or excessive bony growths along the lingual surface of the lower jaw (Figure 1). The tori grow from the alveolar process above the mylohyoid ridge and are composed of periosteal and Haversian tissue (Rouas and Midy, 1997) with trabecular bone in large tori (Seah, 1995). The tori vary in size, number, shape, and location. Mandibular exostoses occur more frequently next to premolars and molars, unilaterally or bilaterally, and consist of single or multiple smooth nodular growths, fused nodules, or continuous ridges. TM is pathological but relatively benign and may hinder dental prosthetics such as dentures and in severe cases affect speech and mastication (Pynn et al., 1995; Sonnier et al., 1999; Bruce et al., 2004; Al Quran and Al-Dwairi, 2006; García-García et al., 2010; Choi et al., 2012).

Although TM has been reported in the fields of dentistry and skeletal biology for decades, there are diverse hypotheses on its etiology. TM frequency differs amongst popula-

\footnotetext{
* Correspondence to: Helen Cho, Department of Anthropology, Davidson College, 209 Ridge Road/Box 6934, Davidson, NC, USA. E-mail: hecho@davidson.edu

Published online 30 November 2017

in J-STAGE (www.jstage.jst.go.jp) DOI: 10.1537/ase.171103
}

tions with a higher prevalence in the circumpolar regions and East Asian ancestries (Ossenberg, 1981; Pechenkina and Benfer, 2002; García-García et al., 2010). The prevalence is greatest in high-latitude populations (Scott et al., 2016) with a secular trend towards decreasing frequency due to biocultural factors (Haugen, 1992; Hosoi et al., 2003; Igarashi, 2016; Scott et al., 2016). While there is a demonstrable north-south cline in Asia, TM frequencies vary between modern and archaeological samples from East and Southeast Asia (Yaacob et al., 1983; Jainkittivong and Langlais, 2000; Pechenkina and Benfer, 2002; Choi et al., 2012). Sex differences further contribute to the variation, with no discernible pattern based on biological sex. In different geographic regions and time periods, females are reported to have a higher prevalence of TM than their male counterparts (Yaacob et al., 1983; Al-Sebaie and Alwrikat, 2011) while the opposite is observed in some studies (Reichart et al., 1988; Eggen and Natvig, 1991; Haugen, 1992; Sonnier et al., 1999; Ihunwo and Phukubye, 2006; Sawair et al., 2009), or no sex differences are reported (Mayhall and Mayhall, 1971; Axelsson and Hedegård, 1981; King and King, 1981; Balaez et al., 1983; Reichart et al., 1988; Eggen, 1992; Pechenkina and Benfer, 2002; Bruce et al., 2004; Al Quran and Al-Dwairi, 2006; Igarashi et al., 2008; Choi et al., 2012).

Additionally, bone responds to mechanical stress and TM may develop from chewing food or paramasticatory func- 

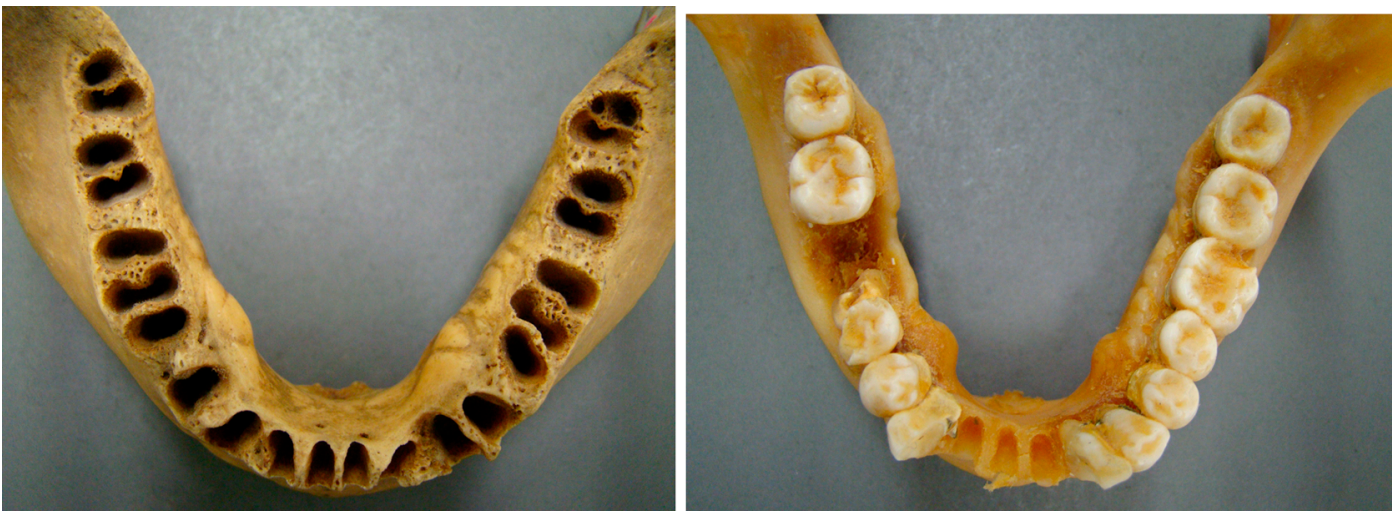

Figure 1. Torus mandibularis (modern Koreans).

tions. Mastication places compressive forces on mandibular teeth, and pressure on the periodontal ligament in the roots causes new bone to form on the alveolar process of the lingual surface (Hylander, 1977; Ossenberg, 1981; Pechenkina and Benfer, 2002). Oral tori are also associated with rough, coarse diets. For example, the Inuit from the Northwest Territories in Canada, one of the circumpolar populations reported to have the highest rates of TM in the world, demonstrate large differences in TM prevalence with dietary change (Mayhall, 1970; Mayhall and Mayhall, 1971). Bruxism, a common type of paramasticatory function, is an intense grinding or clenching of teeth during sleep or during the day, and resulting excessive forces (Clifford et al., 1996; Sirirungrojying and Kerdpon, 1999), temporomandibular joint disorders, or other oral health indicators (Ossenberg, 1981; Pechenkina and Benfer, 2002) may lead to development of exostoses.

Oral tori become apparent in young adulthood during the second and third decades of life (Mayhall and Mayhall, 1971; Balaez et al., 1983; Eggen and Natvig, 1986; Bruce et al., 2004; Igarashi et al., 2008; Al-Sebaie and Alwrikat, 2011; Choi et al., 2012), but they have been observed in preadolescent children and adolescents (Moorrees et al., 1952; Mayhall and Mayhall, 1971; Axelsson and Hedegård, 1981; Eggen and Natvig, 1986; Bruce et al., 2004). The presence of tori at a young age indirectly supports the role of the genes, while an age-related increase in TM frequency is indicative of the alveolar tissue's response to external factors. The latter does not rule out a genetic predisposition, however, as a mechanical stress threshold may have to be reached for its development. While masticatory or paramasticatory stress that accumulates over the years may explain the higher TM prevalence in young to middle-age adults, exostoses may develop as a response to dental attrition in older individuals. Conversely, antemortem tooth loss, alveolar resorption, and thus decreased masticatory stress can lead to lower TM frequency (Ossenberg, 1981; Jainkittivong and Langlais, 2000; Pechenkina and Benfer, 2002). Whether genetic and behavioral factors play a role in tori formation, it is a 'dynamic phenomenon' throughout an individual's lifespan (Ossenberg, 1981).

The common occurrence and interpopulation variation of TM afford an interesting opportunity to examine these oste- ological traits from an anthropological perspective. Given the variation in oral tori by geographic ancestry, sex, mechanical stress, age, and other factors in archaeological and contemporary populations, a comparative approach is suitable to elucidate the etiology. The majority of the literature examines a single population from a specific time period, and the present study is unique in that the comparison of archaeological and modern skeletal populations with biological, cultural, and geographical affinities can contribute to a better understanding of the genetic and environmental influences. The aims of the present study are twofold: (i) to describe the pattern of mandibular tori in the Korean peninsula to corroborate or refute previous studies that report highprevalence statistics among East Asians, and (ii) to compare archaeological and modern skeletal samples from Korea to investigate the secular trend in TM as observed in Nordic (Scott et al., 1991, 2016) and Japanese populations (Igarashi, 2016).

\section{Materials and Methods}

Much of modern Korean society's cultural practices are attributed to the Joseon Dynasty. The Joseon Dynasty lasted from the late 14th to early 20th centuries, the longest dynasty in the history of the peninsula. In addition to cultural affiliation, the Korean population has biological affinity to the Joseon Dynasty. The dynasty remained largely insulated from outside populations and international trade until the late 19th century (Kim, 2009), although the biological relatedness and clustering of East Asian geopolitical nations (Korea, Japan, China) cannot be ignored due to continuous prehistoric and historic events and shifting geographical boundaries (Lee, 2004). Secular changes in Korean stature corroborate the historical records of a relatively insular kingdom as stature increased abruptly at the beginning of the 20th century with the advent of modernization and industrialization in the peninsula (Shin et al., 2012).

Joseon Dynasty skeletal collections from the 15th-18th centuries are housed at the Seoul National University School of Medicine and Ewha Woman's University School of Medicine in Seoul $(n=119$; 56 males, 60 females, 3 indeterminate sex). The Joseon samples were collected from limesoil-mixture-barrier (LSMB) tombs in Seoul and adjacent 
Gyeonggi Province. Although LSMB tombs' high cost limited their use to the upper-class neo-Confucian elites when introduced in Joseon society during the 15th century, lowerclass individuals such as wealthy peasants and the middle class also constructed LSMB tombs in the 17th and 18th centuries (Shin et al., 2008). Therefore, the Joseon sample is assumed to represent individuals from multiple social strata. Modern Korean skeletal samples from the 20th and 21st centuries housed at the Yonsei University School of Dentistry and the Catholic University School of Medicine in Seoul were examined for comparison $(n=77 ; 44$ males, 21 females, 12 indeterminate sex).

For the purposes of the study, a standardized data collection protocol was developed. One of the challenges of investigating mandibular tori is morphological variation. The protocol entailed scoring, describing, and photographing the presence/absence, location, symmetry, and degree of mandibular tori development. A torus has a dimension in the mesiodistal direction, thickness in the lingual direction, and vertical height (Eggen, 1989). Additionally, there is shape variation. Choi et al. (2012) quantified the mandibular tori from CT images, but this was limited to measuring the cortical thickness of each torus, only one of the dimensions observable on dry bone. Due to variation in mandibular exostoses, in the present study, scoring consisted of non-metric recording: presence or absence, unilateral (left or right) or bilateral, solitary or multiple nodules (fused or separate), round or elongated, and location of each nodule (central or lateral incisor, canine, 1st and/or 2nd premolar, 1st and/or 2nd and/or 3rd molar).

Standardized osteological methods for estimating the sex and age-at-death of skeletonized individuals are well established in biological anthropology. For each archaeological skeleton, age-at-death (e.g. morphological changes in the sternal rib, auricular surface, pubic symphysis) and sex (e.g. non-metric traits of the os coxa, cranium, mandible) estimations were carried out, employing methods that were dependent on the state of preservation and presence of relevant skeletal elements and features. Age and sex are documented for most individuals in the modern skeletal collections.

SPSS Statistics, version 24.0 (IBM Corp., New York) was utilized for statistical analyses. A chi-square test was performed to measure the relationships between the categorical variables (Joseon/modern time period, TM presence/absence, male/female sex), the effects of the age cohorts on the TM frequency data, and phi for measures of association between two categorical variables.

\section{Results}

The age and sex distribution of the archaeological and modern samples is summarized in Table 1. Sixteen (13.4\%) Joseon Dynasty individuals out of 119 exhibited TM, and 42 individuals $(54.6 \%)$ out of 77 modern Koreans had TM (Table 2). In 16 Joseon archaeological individuals, the trait is expressed most commonly as round solitary nodules that are adjacent to the premolars; the nodules are seen less frequently next to the canines and uncommon next to the first molars. In 42 modern Koreans with TM, expression of TM mostly exists as round solitary nodules adjacent to premolars
Table 1. Age and sex distribution of the skeletal samples

\begin{tabular}{|c|c|c|c|c|c|c|}
\hline \multirow[b]{2}{*}{ Period } & \multirow[b]{2}{*}{ Sex } & \multicolumn{5}{|c|}{ Age (years) } \\
\hline & & $0-19$ & 20-39 & $40-59$ & $60+$ & $\begin{array}{l}\text { Indeter- } \\
\text { minate }\end{array}$ \\
\hline \multirow[t]{3}{*}{ Joseon } & Female & 2 & 28 & 20 & 3 & 7 \\
\hline & Male & 2 & 16 & 31 & 1 & 6 \\
\hline & Indeterminate & 1 & 0 & 0 & 0 & $2^{\mathrm{a}}$ \\
\hline \multirow[t]{4}{*}{ Modern } & Female & 0 & 0 & 7 & 14 & 0 \\
\hline & Male & 3 & 3 & 22 & 16 & 0 \\
\hline & Indeterminate & 0 & 0 & 0 & 0 & $12^{\mathrm{a}}$ \\
\hline & Total & 8 & 47 & 80 & 34 & 27 \\
\hline
\end{tabular}

${ }^{a}$ Individuals of indeterminate sex and age

Table 2. Chi-square test of Joseon and modern Koreans and torus mandibularis (TM)

\begin{tabular}{lccc}
\hline Period & TM present & TM absent & Total \\
\hline Joseon & 16 & 103 & 119 \\
Modern & 42 & 35 & 77 \\
\hline
\end{tabular}

$\chi^{2}=37.903, \mathrm{df}=1, P=0.000, \varphi=0.440$

Table 3. Chi-square test of sample sizes between sex and time period

\begin{tabular}{lccc}
\hline Period & Female & Male & Total \\
\hline Joseon & 60 & 56 & 116 \\
Modern & 21 & 44 & 65 \\
\hline
\end{tabular}

$\chi^{2}=6.352, \mathrm{df}=1, P=0.012, \varphi=0.187$

and canines with infrequent occurrence next to incisors and first molars. A chi-square test was run to compare frequency data and to measure the relationship between the categorical variables, period (Joseon/modern) and TM (presence/absence). Chi-square is also a test of homogeneity of proportions and tests whether the percentages of individuals with the presence of TM are significantly different between Joseon and modern Koreans. Modern Koreans had a significantly higher frequency of TM than found in Joseon individuals $(54.6 \%$ versus $13.4 \%) \quad\left(\chi^{2} \quad(1, n=196)=37.903\right.$, $P=0.000)$. Phi is a measure of association and also measures effect size; there is a medium to large effect size $(\varphi=0.440, \mathrm{df}=1)$ on the difference in TM between the archaeological and modern samples (Table 2).

Sample distribution by sex is significantly different between the archaeological and modern samples $\left(\chi^{2}(1, n=\right.$ $181)=6.352, P=0.012$ ) (Table 3$)$. Therefore, we checked the TM difference between the samples by individual sex and the TM difference between sexes in each sample. In females, modern Koreans exhibited significantly higher TM frequency than the Joseon females $\left(\chi^{2}(1, n=81)=13.888\right.$, $P=0.000)$ and the modern males also exhibited higher frequency than the Joseon males $\left(\chi^{2}(1, n=100)=14.780\right.$, $P=0.000)$. Both sexes have medium effect sizes (Table 4$)$. There was no sex difference in the Joseon period $\left(\chi^{2}(1\right.$, $n=116)=1.504, P=0.220)$ nor in the modern Koreans $\left(\chi^{2}\right.$ $(1, n=65)=0.273, P=0.601)$ (Table 4).

Lastly, TM frequencies were examined by age categories in the two samples. There were no significant differences of 
Table 4. Torus mandibularis frequencies by sex and the two samples of Joseon and modern Koreans

\begin{tabular}{lccccc}
\hline \multirow{2}{*}{ Period } & \multicolumn{2}{c}{ Female } & & \multicolumn{2}{c}{ Male } \\
\cline { 2 - 3 } \cline { 5 - 6 } & TM present & TM absent & & TM present & TM absent \\
\hline Joseon & 6 & 54 & & 10 & 46 \\
Modern & 10 & 11 & & 24 & 20 \\
\hline
\end{tabular}

Joseon vs. modern female $\chi^{2}=13.888, \mathrm{df}=1, P=0.000, \varphi=0.414$

Joseon vs. modern male $\chi^{2}=14.780, \mathrm{df}=1, P=0.000, \varphi=0.384$

Joseon female vs. male $\chi^{2}=1.504, \mathrm{df}=1, P=0.220$

Modern female vs. male $\chi^{2}=0.273, \mathrm{df}=1, P=0.601$

Table 5. Torus mandibularis frequencies of Joseon and modern Koreans by age categories

\begin{tabular}{lccccc}
\hline \multirow{2}{*}{ Age } & \multicolumn{2}{c}{ Joseon } & & \multicolumn{2}{c}{ Modern } \\
\cline { 2 - 3 } \cline { 5 - 6 } & TM present & TM absent & & TM present & TM absent \\
\hline $0-19$ & 0 & 5 & & 2 & 1 \\
$20-39$ & 6 & 38 & & 2 & 1 \\
$40-59$ & 6 & 45 & & 12 & 17 \\
$60+$ & 1 & 3 & & 18 & 12 \\
\hline
\end{tabular}

Joseon $\chi^{2}=1.363, \mathrm{df}=3, P=0.714$

Modern $\chi^{2}=2.596, \mathrm{df}=3, P=0.458$

TM frequencies between the age categories in the Joseon $\left(\chi^{2}\right.$ $(3, n=104)=1.363, P=0.714)$ or in the modern Koreans $\left(\chi^{2}(3, n=65)=2.596, P=0.458\right)($ Table 5$)$.

\section{Discussion}

The general trend across the world is towards a reduction in the occurrence of TM (Ossenberg, 1981). In a study of the Inuit (Mayhall, 1970), villagers who underwent cultural and dietary changes demonstrated a decrease in TM prevalence (47\% females, $28 \%$ males) compared to $62 \%$ females and $54 \%$ males who maintained a native diet. Similarly, there is a significant decrease in TM frequency from $87 \%$ in the Japanese Jomon era (12000-3000 BP) to $66 \%$ in the Yayoi era $(3000-1750 \mathrm{BP})$ that is attributed to the admixture of Yayoi with other populations from continental Asia and/or a decreased occlusal stress from a harder nut-based staple to a softer rice-based diet. The trend continued and a study of modern Japanese women further demonstrates that the secular trend is towards a decreasing prevalence in the archipelago (Hosoi et al., 2003). There was also a decrease in TM prevalence from $100 \%$ to $48 \%$ over several hundred years in the North Atlantic among the medieval Norse and a reduction from large to small tori expression (Scott et al., 1991). The present study, however, yielded inconsistent results. There is a significant increase in TM frequency from the Joseon Dynasty (13.3\%) to modern Koreans (54.6\%) in both sexes, perhaps indicative of some external variables that have changed over the centuries.

To reveal multifactorial causes in TM development in Joseon and modern Korean society, we considered the interaction of genetic inheritance and lifestyle factors. As TM prevalence is similar between females and males among the Joseon and modern Koreans, the causal factors appear to affect both sexes. We tried first to posit the role of mechanical stress in TM frequency with the detailed evidence for changes in masticatory stress from the Joseon to modern Korean periods. Masticatory or parafunctional stress that accumulates over the years and leads to the development of the exostoses necessitates an age-related increase in TM prevalence. In the present study, Joseon and modern samples exhibit unequal age distributions across the four age categories with very few young individuals. Thus, the pattern of TM is greater representation in the middle two cohorts in Joseon and latter two cohorts in modern samples. As statistical analysis demonstrates no age effect in either of the samples and a larger number of individuals across the age groups is necessary to observe possible age-related trends, we cannot draw definitive conclusions or identify the extrinsic factors to support the mechanical stress threshold model.

Concerning the role of a dietary shift in TM prevalence, the Joseon and modern Korean diets are not dramatically different, and not characterized as particularly coarse or tough but remained relatively soft throughout the centuries. Although the staple may have been a mix with millet, barley, or other grains depending on the individual's social class and geographic region, Koreans have long been eating the main staple of cooked rice with side dishes of cooked, fresh, and pickled vegetables, soup, and dried fish (Park, 1992; Chung, 2014). The secular trend in TM prevalence thus cannot be attributed to notable dietary changes in the Korean peninsula as is the case with the Inuit from the Canadian Northwest Territories (Mayhall, 1970), prehistoric and historic Japanese (Hosoi et al., 2003; Igarashi, 2016), and the medieval period Norse from Scandinavia (Scott et al., 1991; Haugen, 1992).

Rather, the present study might support the quasicontinuous model with environmental factors in the development of oral tori (Ossenberg, 1981; Eggen, 1989; Haugen, 1992). In a comparative study of medieval Norse and 14th-17th-century Inuit from Greenland occupying similar physical environments, the Norse TM are more extreme, longer, and irregularly shaped, whereas the Inuit tori are weakly developed and smoother. Sellevold (1980) concludes that the two populations exhibit different phenotypes of the same genetic trait. Likewise, TM in the Joseon period and modern Koreans are similar in shape and type, and the round solitary nodule type may be due to their shared biology. In the Joseon mandibles, TM occurs next to premolars, whereas in the modern mandibles, TM is found next to premolars and canines. This variation in location may be due to secular changes in skull morphology or other variables not identified in the present study. Our results cannot suggest or disprove the existence of an underlying hereditary component for TM in ancient and modern Koreans. Direct evidence is necessary through genetic or pedigree analyses to understand the characteristics of TM in the Korean peninsula.

$\mathrm{TM}$ is common in past and present populations from diverse geographical locations, and a few studies on oral exostoses have investigated populations with biological and geographical affinity for secular trends (Scott et al., 1991, 2016; Igarashi, 2016). The results of our study of TM in Joseon and modern Korean people are generally consistent with the literature, which reports a higher frequency in Asiatic populations, but are contrary to the worldwide trend of decreasing prevalence over time. As we cannot draw conclusions to 
support specific causal factors responsible for the development of TM, future studies should test the genetic role, effects of mandibular robusticity, and age-related attrition in the occurrence of the tori. TM is a dynamic phenomenon in the lifetime of an individual, and perhaps a longitudinal study of contemporary people through CT imaging could be employed to investigate the development of TM over the decades.

\section{Acknowledgments}

We thank Dr. Eun-jin Woo from the College of Dentistry at Yonsei University for assistance with statistical analysis.

\section{References}

Al Quran F.A.M. and Al-Dwairi Z.N. (2006) Torus palatinus and torus mandibularis in edentulous patients. Journal of Contemporary Dental Practice, 7: 112-119.

Al-Sabaie D. and Alwrikat M. (2011) Prevalence of torus palatinus and torus mandibularis in Jordanian population. Pakistan Oral and Dental Journal, 31: 214-216.

Axelsson G. and Hedegård B. (1981) Torus mandibularis among Icelanders. American Journal of Physical Anthropology, 54: 383-389.

Balaez A.B., Diaz E.M., and Perez I.R. (1983) Prevalencia de torus palatinos y mandibulares en Ciudad de La Habana. Revista Cubana de Estomalogía, 20: 126-132.

Bruce I., Ndanu T.A., and Addo M.E. (2004) Epidemiological aspects of oral tori in a Ghanaian community. International Dental Journal, 54: 78-82.

Choi Y., Park H., Lee J.S., Park J.C., Kim C.S., Choi S.H., Cho K.S., Chai J.K., and Jung U.W. (2012) Prevalence and anatomic topography of mandibular tori: computed tomographic analysis. Journal of Oral and Maxillofacial Surgery, 70: 1286-1291.

Chung Y. (2014) Eating culture. In: Shin M.D. (ed.), Everyday Life in Joseon-Era Korea: Economy and Society. Leiden, Global Oriental, pp. 225-234.

Clifford T., Lamey P.J., and Fartash L. (1996) Mandibular tori, migraine and temporomandibular disorders. British Dental Journal, 180: 382-384.

Eggen S. (1989) Torus mandibularis: an estimation of the degree of genetic determination. Acta Odontologica Scandinavica, 47: 409-415.

Eggen S. (1992) Correlated characteristics of the jaws: association between torus mandibularis and marginal alveolar bone height. Acta Odontologica Scandinavica, 50: 1-6.

Eggen S. and Natvig B. (1986) Relationship between torus mandibularis and number of present teeth. Scandinavian Journal of Dental Research, 94: 233-240.

Eggen S. and Natvig B. (1991) Variation in torus mandibularis prevalence in Norway. Community Dentistry and Oral Epidemiology, 19: 32-35.

García-García A.S., Martínez-González J., Gómez-Font R., SotoRivadeneira A., and Oviedo-Roldán L. (2010) Current status of the torus palatinus and torus mandibularis. Medicina Oral Patologia Oral y Cirugia Bucal, 15: 353-360.

Haugen L.K. (1992) Palatine and mandibular tori: a morphologic study in the current Norwegian population. Acta Odontologica Scandinavica, 50: 65-77.

Hosoi T., Yoda T., Yamaguchi M., Amano H., and Orimo H. (2003) Elderly women with oral exostoses had higher bone mineral density. Journal of Bone and Mineral Metabolism, 21: 120122.

Hylander W.L. (1977) The adaptive significance of Eskimo craniofacial morphology. In: Dahlberg A.A. and Graber T.M. (eds.),
Orofacial Growth and Development. Mouton, The Hague, pp. 129-169.

Igarashi Y. (2016) Frequency of mandibular tori in prehistoric and historic Japanese island populations. Quaternary International, 405: 87-100.

Igarashi Y., Ohzeki S., Uesu K., Nakabayashi T., and Kanazawa E. (2008) Frequency of mandibular tori in the present-day Japanese. Anthropological Science, 116: 17-32.

Ihunwo A.O. and Phukubye P. (2006) The frequency and anatomical features of torus mandibularis in a Black South African population. Homo, 57: 253-262.

Jainkittivong A. and Langlais R.P. (2000) Buccal and palatal exostoses: prevalence and concurrence with tori. Oral surgery, Oral Medicine, Oral Pathology, Oral Radiology, and Endodontics, 90: 48-53.

Kim H. (2009) Chosun as seen by Westerners (Series No. 2): From the opening of Korea to the outbreak of the Sino-Japanese war. Journal of Korean Politics, 18: 247-314 (in Korean).

King D.R. and King A.C. (1981) Incidence of tori in three population groups. Journal of Oral Medicine, 36: 21-23.

Lee J. (2004) The relatedness between the origin of Japanese and Korean ethnicity. Doctoral dissertation, The Florida State University.

Mayhall J.T. (1970) The effect of culture change upon the Eskimo dentition. Arctic Anthropology, 7: 117-121.

Mayhall J.T. and Mayhall M.F. (1971) Torus mandibularis in two Northwest Territories villages. American Journal of Physical Anthropology, 34: 143-148.

Moorrees C.F.A., Osborne R.H., and Wilde E. (1952) Torus mandibularis: its occurrence in Aleut children and its genetic determinants. American Journal of Physical Anthropology, 10: 319-330.

Ossenberg N.S. (1981) Mandibular torus: a synthesis of new and previously reported data, and a discussion of its cause. In: Cybulski J.S. (ed.), Contributions to Physical Anthropology, 1978-1980. National Museum of Canada, Ottawa, pp. 1-52.

Park S. (1992) The eating and cooking spaces of Yang-ban houses in the Cho-sun dynasty. Journal of Architectural History, 1: 39-51 (in Korean).

Pechenkina E.A. and Benfer R.A., Jr (2002) The role of occlusal stress and gingival infection in the formation of exostoses on mandible and maxilla from Neolithic China. Homo, 53: 112130 .

Pynn B.R., Kurys-Kos N.S., Walker D.A., and Mayhall J.T. (1995) Tori mandibularis: a case report and review of the literature. Journal of the Canadian Dental Association, 61: 1057-1066.

Reichart P.A., Neuhaus F., and Sookasem M. (1988) Prevalence of torus palatinus and torus mandibularis in Germans and Thai. Community Dentistry and Oral Epidemiology, 16: 61-64.

Rouas A. and Midy D. (1997) About a mandibular hyperostosis: the torus mandibularis. Surgical and Radiologic Anatomy, 19: $41-43$.

Sawair F.A., Shayyab M.H., Al-Rabab'ah M.A., and Saku T. (2009) Prevalence and clinical characteristics of tori and jaw exostoses in a teaching hospital in Jordan. Saudi Medical Journal, 30: 1557-1562.

Scott G.R., Halffman C.M., and Pedersen P.O. (1991) Dental conditions of medieval Norsemen in the North Atlantic. Acta Archaeologica, 62: 183-207.

Scott G.R., Schomberg R., Swenson V., Adams D., and Pilloud M.A. (2016) Northern exposure: mandibular torus in the Greenlandic Norse and the whole wide world. American Journal of Physical Anthropology, 161: 513-521.

Seah Y.H. (1995) Torus palatinus and torus mandibularis: a review of the literature. Australian Dental Journal, 40: 318-321.

Sellevold B.J. (1980) Mandibular torus morphology. American Journal of Physical Anthropology, 53: 569-572.

Shin D.H., Oh C.S., Kim Y.-S., and Hwang Y.-I. (2012) Ancient-to-modern secular changes in Korean stature. American Journal of Physical Anthropology, 147: 433-442. 
Shin M.H., Yi Y.S., Bok G.D., Lee E.J., Spigelman M., Park J.B., Min S.R., and Shin D.H. (2008) How did mummification occur in bodies buried in tombs with a lime soil mixture barrier during the Joseon Dynasty in Korea. In: Peña P.A., Martin C.R., Rodríguez A.R., and Aufderheide A.C. (eds.), Mummies and Science, World Mummy Research: Proceedings of the VI World Congress on Mummy Studies, Teguise, Lanzarote. Academia Canaria de la Historia, Santa Cruz de Tenerife, pp. $105-113$.
Sirirungrojying S. and Kerdpon D. (1999) Relationship between oral tori and temporomandibular disorders. International Dental Journal, 49: 101-104.

Sonnier K.E., Horning G.M., and Cohen M.E. (1999) Palatal tubercles, palatal tori, and mandibular tori: prevalence and anatomical features in a US population. Journal of Periodontology, 70: 329-336.

Yaacob H., Tirmizi H., and Ismail K. (1983) The prevalence of oral tori in Malaysians. Journal of Oral Medicine, 38: 40-42. 